Abanico Veterinario. Enero-Diciembre 2021; 11:1-16. http://dx.doi.org/10.21929/abavet2021.27

Revisión de Literatura. Recibido: 18/01/2021. Aceptado: 02/06/2021. Publicado: 10/06/2021. Clave: e2021-6.

\title{
Revisión sistemática de las diferentes técnicas quirúrgicas de contracepción en gatas
}

Systematic review of different surgical contraception techniques in queens

\author{
Toledo-Valdez Cielo*11D , Rivera-Barreno Ramón ${ }^{1 \mathrm{ID}}$, Talamantes-Lima IIse ${ }^{1 \text { ID, }}$ \\ Bustos-Varela Jocelyn ${ }^{11 D}$, García-Herrera Ricardo ${ }^{21 D}$, Rodríguez-Alarcón Carlos ${ }^{\star * 11 D}$
}

1Departamento de Ciencias Veterinarias. Universidad Autónoma de Ciudad Juárez. Chihuahua, México. 2División Académica de Ciencias Agropecuarias, Universidad Juárez Autónoma de Tabasco. Villahermosa, Tabasco, México. *Autor responsable: Cielo Toledo-Valdez. ${ }^{* *}$ Autor de correspondencia: Carlos Rodríguez Alarcón. Departamento de Ciencias Veterinarias. Universidad Autónoma de Ciudad Juárez, Av. Benjamín Franklin No. 4650, Zona PRONAF, 32315, Ciudad Juárez, Chihuahua., México. Tel. +526566881800. E-mail: cielotoledo90@gmail.com, rrivera@uacj.mx, al144450@alumnos.uacj.mx, jocelyn2202@hotmail.com, ricardogarciaherrera@hotmail.com, carrodri@uacj.mx

\section{RESUMEN}

En la clínica veterinaria, la cirugía contraceptiva es la más practicada en gatas. Sin embargo, no está establecido cuál técnica quirúrgica es la más adecuada. Con la finalidad disminuir el dolor posoperatorio, tiempo de recuperación, tiempo de cirugía y facilidad técnica para el cirujano, se han descrito diferentes técnicas quirúrgicas y variaciones de las mismas. Esto ha creado el dilema sobre la realización de ovariohisterectomía contra la ovariectomía; tipo de abordaje: línea media, lateral o laparoscópico; y método de ligadura del pedículo ovárico: bisturí harmónico, clips de plástico y titanio, electrocauterio bipolar, nudo pedicular y ligadura tradicional. Teniendo esto en cuenta, con el objetivo de establecer cuál es la cirugía contraceptiva más adecuada en gatas y apoyados en la medicina basada en evidencias, se realizó una revisión sistemática. Utilizando los buscadores Google Académico, Web of Science, PubMed y Scielo, con las siguientes variables a evaluar: dolor, tiempo y dificultad técnica. Se encontró que existe poca evidencia para establecer la superioridad de alguna técnica quirúrgica sobre otra y que la elección de la técnica quirúrgica debe de ser realizada en base al paciente, habilidades del cirujano y la disponibilidad de materiales.

Palabras claves: contracepción, gatas, ovariohisterectomía, ovariectomía, abordajes quirúrgicos.

\begin{abstract}
In the veterinary clinic, contraceptive surgery is the most commonly performed surgery in female cats. However, it is not established which surgical technique is the most appropriate. In order to reduce postoperative pain, recovery time, surgery time and technical ease for the surgeon, different surgical techniques and variations of these techniques have been described. This has created the dilemma of performing ovariohysterectomy versus oophorectomy; type of approach: midline, lateral or laparoscopic; and method of ovarian pedicle ligation: harmonic scalpel, plastic and titanium clips, bipolar electrocautery, pedicle knot and traditional ligation. With this in mind, with the objective of establishing which is the most adequate contraceptive surgery in female cats and supported by evidence-based medicine, a systematic review was carried out. Using Google Scholar, Web of Science, PubMed and Scielo search engines, with the following variables to be evaluated: pain, time and technical difficulty. It was found that there is little evidence to establish the superiority of any surgical technique over another and that the choice of surgical technique should be made based on the patient, the surgeon's skills and the availability of materials.
\end{abstract}

Keywords: Contraception, cats, ovariohysterectomy, ovariectomy, surgical approaches. 


\section{INTRODUCCIÓN}

Los procedimientos quirúrgicos de contracepción son la primera opción para evitar la reproducción en gatas en el mundo. Se han establecido dos factores fundamentales para justificar el control reproductivo de los gatos. El primero es la importancia misma de controlar la sobrepoblación de esta especie; y el segundo es la prevención de algunas enfermedades del tracto reproductivo y glándula mamaria (Overley et al., 2005). A través del tiempo, se han descrito dos técnicas quirúrgicas para la contracepción en gatas, la ovariohisterectomía $(\mathrm{OVH})$ que se define como la remoción completa del tracto reproductor y la ovarioectomía (OV) que consiste en la eliminación exclusiva de los ovarios (Howe 2006; Pereira et al., 2018). Ambas técnicas son igualmente efectivas para controlar la población de la especie y en reducir la posibilidad de presencia de tumores de glándula mamaria cuando se practican en edades tempranas (Frasson, 2018).

La cirugía contraceptiva representa un alto porcentaje de los procedimientos que se realizan en gatas. Con la idea de reducir el dolor, la dificultad, tiempo y costo de estas cirugías se han desarrollado variantes en las últimas décadas (Griffin et al., 2016). Las principales variantes ocurren en el tipo de ligadura empleada en los pedículos ováricos. Los diferentes tipos son : ligadura mediante el uso de una o dos pinzas, el uso único de ligadura con sutura, clips hemostáticos, equipos de electrocirugía monopolares, bipolares o a base de ultrasonido (Boursier et al., 2018; Guedes et al., 2017; Miller et al., 2016). Básicamente existen dos abordajes para efectuar tanto la OVH como la OV en gatas: el abordaje por línea media también conocido como celiotomía y el abordaje lateral o por el flanco. En los Estados Unidos, la mayoría de los veterinarios prefieren el abordaje por celiotomía, mientras que en Europa es preferida la técnica lateral (Bushby y White, 2019; Cuddy, 2016). Recientemente, se ha tomado a la laparoscopia como una alternativa viable. Esta técnica mínimamente invasiva permite una mejor visión de las estructuras, así como una disminución de riesgos y dolor posoperatorio, acortando también los tiempos de cirugía y de recuperación del paciente (Da Conceição et al., 2018; Howe, 2006; Phypers, 2017).

Por lo tanto, existen diversas técnicas quirúrgicas en la contracepción en gatas, en las cuales cada cirujano describe las ventajas y bondades de los abordajes o métodos hemostáticos. Sin embargo, no se ha establecido de manera concreta cual es la más apropiada en esta especie, pues en la literatura científica escasea la información sobre cual técnica causa menos dolor, requiere menos tiempo o es técnicamente más fácil de realizar. Es por eso que, con el objetivo de identificar las ventajas y desventajas de estas técnicas quirúrgicas, se realizó una revisión sistemática para valorar su beneficio y manifestar cuál de ellas es más segura, rápida y menos dolorosas para la gata. 


\section{MATERIAL Y MÉTODOS}

Para la realización de este trabajo se revisó la literatura publicada, en los buscadores científicos Google Académico, PubMed y Web of Science, y se emplearon los siguientes criterios de búsqueda: spay, cats, ovariohistectomy, ovarioectomy, contraception, surgery, techniques, laparotomy en los idiomas inglés, español y portugués.

Se incluyeron estudios de los años 2006 al 2020, en los cuales se describiera una técnica quirúrgica de contracepción en gatas. Se analizaron estudios que evaluaban los riesgos, beneficios e indicaciones de la técnica quirúrgica en particular, con énfasis en las que discutían y se comparaban con alguna otra. También se incluyeron revisiones de literatura y reportes de casos clínicos de diferentes técnicas quirúrgicas de contracepción en gatas.

Todos los estudios incluidos fueron categorizados de acuerdo con a la clasificación de la evidencia científica establecida por Sackett y Wennberg (1997). Está clasificación cuenta con cinco niveles de estratificación, los cuales han sido avalados por el Canadian Task Force on Preventive Health Care quienes fueron los primeros en determinar los niveles de evidencia que un estudio científico ofrece (Manterola et al., 2014).

Una vez clasificados los artículos, se analizaron para la sección de discusión aquellos agrupados en los niveles III al I. Las variables a discutir en este manuscrito son: tiempo de cirugía, dolor tanto trans como posquirúrgico, dificultad técnica de la cirugía y tiempo de aprendizaje o dificultad técnica de cada una.

\section{RESULTADOS Y DISCUSIÓN}

El resultado original de la búsqueda fueron 6760 publicaciones. Después de descartar todos los estudios realizados en otra especie, capítulos de libro o publicaciones donde no se evaluó la técnica quirúrgica y con base en la clasificación de la evidencia científica, se obtuvieron 31 investigaciones distribuidas en las cinco categorías existentes según Manterola et al. (2014) (cuadro 1). 
Cuadro 1. Representación de los niveles de la pirámide de la evidencia y la agrupación de los estudios en cada nivel

\begin{tabular}{|c|c|}
\hline ivel & \\
\hline $\begin{array}{l}\text { Nivel } \\
\text { I }\end{array}$ & $\begin{array}{l}\text { Howe LM. (2006). Surgical methods of contraception and sterilization. Theriogenology. } \\
\text { - } \quad \text { Sakals SA et al., (2018). Evaluation of laparoscopically assisted ovariectomy technique in cats. Veterinary surgery. } \\
\text { - } \quad \text { Swaffield MJ, Molloy SL, Lipscomb VJ. (2020). Prospective comparison of perioperative wound and pain score } \\
\text { parameters in cats undergoing flank vs midline ovariectomy. Journal of Feline Medicine and Surgery }\end{array}$ \\
\hline $\begin{array}{l}\text { Nivel } \\
\text { II }\end{array}$ & 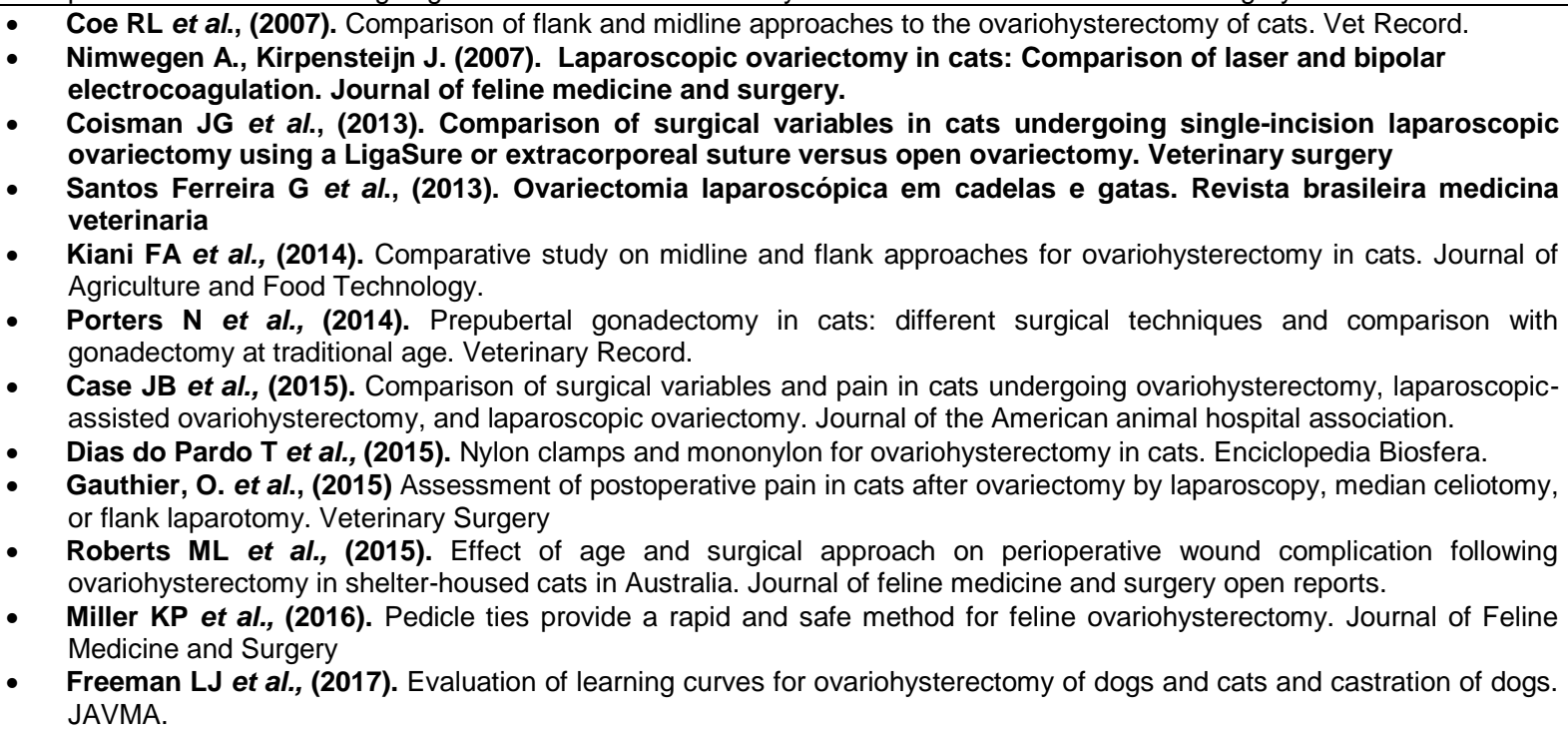 \\
\hline $\begin{array}{l}\text { Nivel } \\
\text { III }\end{array}$ & $\begin{array}{l}\text { - Burrow R et al., (2006). Prospective evaluation of postoperative pain in cats undergoing ovariohisterectomy by mindline } \\
\text { or flank approach. Vet Record. } \\
\text { - Grint N et al., (2006) Assessment of the influence of surgical technique on postoperative pain and wound tenderness in } \\
\text { cats following ovariohysterectomy. Journal of feline medicine and surgery. } \\
\text { - } \quad \text { Ferreira MP et al., (2011). Laparoscopic Ovariohysterectomy in Domestic Cats: Two Portals Technique. Acta Scientiae } \\
\text { Veterinari. } \\
\text { Pecere Oliveira J et al., (2014). Pain assessment in cats undergoing ovariohysterectomy by midline or lateral celiotomy } \\
\text { through use of a previously validated multidimensional omposite pain scale. Acta Cirurgica Brasileira } \\
\text { - Tavares DC et al., (2016). Video-assisted ovariohysterectomy in domestic cats (Felis catus, Linnaeus, 1758) using two } \\
\text { access portals. Acta Cirurgica Brasileira. } \\
\text { - Da Costa D et al.,. (2017). LESS ovariohysterectomy in cats using a new homemade multiport. Ciencia Rural. } \\
\text { - Moutinho da Conceicao ME et al., (2017). Description and Executability of a Novel Pre-tied Mini Ligature (Miniloop) in } \\
\text { Laparocopic Ovariectomy in Cats. Acta Scientiae Veterinariae. } \\
\text { Broursier JF et al., (2018). Effectiveness of a bipolar vessel sealant device for ovariohysterectomy in cats with } \\
\text { pyometra. Journal of Feline medicine and Surgery. }\end{array}$ \\
\hline $\begin{array}{l}\text { Nivel } \\
\text { IV }\end{array}$ & $\begin{array}{l}\text { - } \quad \text { Ball RL et al., (2010). Ovarian remnant syndrome in dogs and cats: } 21 \text { cases (2000-2007). JAVMA. } \\
\text { - } \quad \text { Fransson BA., Ragle CA. (2011). Lift laparoscopy in dogs and cats: } 12 \text { cases (2008-2009). JAVMA } \\
\text { - } \quad \text { Lawall T et al., (2017). Minilaparoscopic ovariohysterectomy in healthy cats. Ciencia rural. } \\
\text { Minto BW et al., (2017). Spay hook minimally invasive ovariohysterectomy in cats. Retrospective study of } 276 \text { cases. } \\
\text { Investigação. }\end{array}$ \\
\hline $\begin{array}{l}\text { Nivel } \\
\mathrm{V}\end{array}$ & $\begin{array}{l}\text { - McKenzie B. (2010). Evaluating the benefits and risk of neutering dogs and cats. CAB reviews: Perspectives in } \\
\text { agriculture, veterinary science, nutrition and natural resources } \\
\text { - De Tora M y McCarthy RJ. (2011). Ovariohysterectomy versus ovariectomy for elective sterilization of female dogs and } \\
\text { cats: is removal of the uterus necessary? JAVMA. } \\
\text { Sparkes A. (2011). Neutering cats- Assessing attitudes and challenging conventions. Journal of feline medicine and } \\
\text { surgery. } \\
\text { Howe LM. (2015). Current perspectives on the optimal age to spay/castrate dogs and cats. Veterinary Medicine: research } \\
\text { and reports. } \\
\text { Griffin B et al., (2016). The Association of Shelter Veterinarians' } 2016 \text { Veterinary Medical Care Guidelines for Spay- } \\
\text { Neuter Programs. JAVMA. } \\
\text { Yates D y Goetz U. (2016). Flank or midline ovariohysterectomy in the cat? Companion Animal. }\end{array}$ \\
\hline
\end{tabular}


Diferentes técnicas quirúrgicas de contracepción en gatas, su abordaje y hemostasia.

En gatas está descrito que tanto la OVH como la OV son igualmente benéficas como técnicas quirúrgicas contraceptivas electivas, pero se considera que la longitud de incisión de disminuye en la OV debido a que no es necesaria la exposición del cuerpo del útero (DeTora y McCarthy, 2011; Muraro y White, 2014; Peeters y Kirpensteijn, 2011). Sin embargo, El tiempo de cirugía es usualmente el mismo y los signos de dolor medidos por escala de Glasgow son iguales en ambos procedimientos (Peeters y Kirpensteijn, 2011).

En casos de que exista enfermedad uterina se debe realizar siempre la $\mathrm{OVH}$. Estas dos técnicas quirúrgicas pueden realizarse por abordaje de la línea media o la técnica lateral (Bushby y White, 2019; Stavisky y Brennan, 2020).

\section{Abordaje por línea media}

El abordaje por línea media o celiotomía, es el que tradicionalmente se enseña en las universidades. Aunque técnicamente la palabra celiotomía se refiere a la incisión que abarca desde el cartílago xifoides hasta el pubis, para realizar la $\mathrm{OVH}$ en gatas se incide dos centímetros caudal a la cicatriz umbilical y se extiende en dirección al pubis (Frasson, 2018). Al entrar a cavidad abdominal, se localizan los ovarios con ayuda de un ovariotomo o utilizando el dedo índice. Para liberar un poco más el ovario, se rasga digitalmente el ligamento suspensorio, el cual se inserta caudal y ventral a la última costilla (Hill y Smeak, 2010; McGrath et al., 2004; Yates y Goetz, 2015). Una vez exteriorizado el ovario, se hace hemostasia en el pedículo ovárico, para lo cual existen diversas técnicas (descritas en otra sección).

\section{Abordaje lateral}

El abordaje lateral se utiliza tanto para para realizar OVH y OV y es, aparentemente más sencillo que el abordaje por celiotomía. Este abordaje, en un principio fue indicado en gatas que presentaban un desarrollo excesivo de las glándulas mamarias o en gatas ferales en las cual el cuidado y observación posoperatorio representa una complicación por el manejo (McGrath et al., 2004; Reece, 2018). Se debe tener en cuenta la anatomía de la gata y considerar que el ovario izquierdo esta caudal al riñón, ligeramente ventral a las tercera y cuarta vértebras lumbares. En esta técnica se coloca al animal en decúbito lateral ya sea izquierdo o derecho. Posteriormente, entre 1 y $5 \mathrm{~cm}$ caudal a la última costilla y por debajo de las apófisis transversas lumbares, se realiza una incisión en piel de una longitud de 1 a $4 \mathrm{~cm}$ en dirección dorsoventral. El tejido subcutáneo se incide y se realiza disección roma, al igual que en la fascia muscular conformada por los músculos oblicuo externo, oblicuo interno y el transverso abdominal. Al incidir esté ultimo músculo, se penetra a la cavidad abdominal a través del peritoneo. Inmediatamente se ubica el ovario izquierdo o derecho, se pinza el pedículo ovárico con 
pinzas hemostáticas y se realiza su ligadura. Antes de cortar el pedículo, se localiza el ovario contrario, para lo cual, se ubica la bifurcación uterina (Howe 2006; McGrath et al., 2004; Yates Goetz, 2015), se realiza el vencimiento del ligamento suspensorio y el ovario y se exterioriza a través de la incisión de la misma manera que en el abordaje de la línea media ventral (Kiani et al., 2014), para llevar a cabo el mismo procedimiento que en el primer ovario. Por último, se cierra fascia muscular abarcando los tres músculos en un plano, seguidos de una sutura subcuticular y piel (Howe 2006; SilvaMolano et al., 2007).

\section{Hemostasia del pedículo ovárico}

Una parte fundamental de la cirugía contraceptiva en pequeñas especies es la hemostasia del pedículo ovárico, ya que es el paso en el cual se presentan más complicaciones quirúrgicas, pues su falla conlleva a una hemorragia (Adin, 2011; Bohling, 2020; Pollari et al., 1996; Voorwald et al., 2013). Además, es uno de los pasos más difíciles de realizar de manera adecuada, aun por cirujanos inexpertos (Bushby y White, 2019; Miller et al,. 2016).

En gatas, se puede ligar el pedículo ovárico ayudado con dos pinzas. Al utilizar dos pinzas para realizar la ligadura, estas deben de ir acompañada de 1 o 2 vueltas a la ligadura que circulen al pedículo o una ligadura de transfixión por debajo de la primera pinza (Frasson, 2018; Hill y Smeak, 2010). También se puede hacer con la ayuda de una sola pinza sobre el pedículo ovárico, la cual se recomienda en casos en los que el tracto reproductivo sea pequeño, friable o frágil y no soporte el trauma causado por las dos pinzas (Mayhew y Brown, 2007). El uso de una doble ligadura que circule el pedículo es suficiente en estas gatas y solamente en caso que la gata se encuentre preñada o en estro, sería necesario poner una ligadura de transfixión (Frasson, 2018).

Otra manera de realizar la hemostasia es mediante el empleo del nudo pedicular, que consiste en ligar el pedículo ovárico sobre sí mismo. Este procedimiento se realiza de manera similar al cordón espermático durante la orquiectomía de gatos machos (Howe, 2006; Miller et al., 2016). Aparentemente la principal ventaja de realizar el nudo pedicular, es que permite que la ligadura se realice en menor tiempo, en comparación con la colocación de dos ligaduras sobre la misma estructura (Porters et al., 2014), lo que permite efectuar esta maniobra con un menor tiempo, con disminución del uso de material de sutura y por ende se deja menos o ningún material extraño en el paciente (Bushby y White, 2019; Miller et al., 2016).

De igual forma está descrito que una sola ligadura que abarque el cuerno uterino y el pedículo produce una hemostasia segura y rápida en gatas (Begum y Bhuvaneshwari, 2018). La introducción de nuevas técnicas hemostáticas como el electrobisturi bipolar, también se ha implementado en la cirugía contraceptiva en gatas. Este dispositivo ha mostrado ser efectivo para producir hemostasia en la OVH en gatas (Watts, 2018). 
Discusión del tiempo de cirugía

Aunque pueda pensarse que el tiempo de cirugía no es un factor importante, este debe ser tomado en cuenta como un parámetro significativo cuando se trata de realizar cirugías masivas para el control de reproducción en gatas.

\section{Comparación de tiempo de cirugía en técnicas abiertas}

En un estudio compararon las técnicas de OVH y OV en gatas. Ambas cirugías fueron realizadas por línea media y practicadas por un cirujano experimentado. Los autores encontraron que el tiempo invertido en la realización de la OV, fue significativamente menor al de las OVH (Pereira et al., 2018). En otro estudio, compararon los tiempos de cirugía invertidos con las técnicas abiertas. Estos autores describen que realizar la OVH en gatas pre púberes es significativamente más rápido que cuando se practica en gatas mayores a 8 meses (Porters et al., 2014). Además, estos mismos autores encontraron que el tiempo de cirugía fue menor cuando se utilizó electrocoagulación monopolar, seguido de aplicación de grapas, lazada del pedículo (nudo pedicular) y ligadura.

Por otra parte, en otra investigación encontraron que el uso de nudo pedicular ovárico resultó en un menor tiempo (5 minutos) en comparación con la técnica de nudo con sutura (7 minutos); este tiempo se midió solamente para la hemostasia de pedículo ovárico (Miller et al., 2016).

En un estudio de comparación de abordaje por línea media y flanco lateral, se encontró que los procedimientos por flanco lateral tenían un tiempo promedio de 23 minutos, el cual fue considerablemente menor al registrado en abordaje por línea media (31 minutos) (Kiani et al., 2014). Sin embargo, estos resultados contrastan con lo descrito por otros investigadores, quienes no encontraron diferencias estadísticamente significativas en el tiempo quirúrgico entre estos dos abordajes (Swaffield et al., 2020). Por otro lado, se describe que el tiempo durante la incisión de la piel y la entrada al peritoneo es mayor en el abordaje por flanco lateral, mientras que la localización del útero es más tardada en el abordaje por línea media (Coe et al., 2006).

En un estudio retrospectivo se demostró que el uso de un dispositivo bipolar es seguro y efectivo para realizar OV por celiotomía en gatas. Aunque demostraron que el tiempo de cirugía con esta técnica fue menor en perras, esto no ocurrió en gatas (Watts, 2018). Por último, está definido que la técnica quirúrgica en la que se invierte menos tiempo para realizar la $\mathrm{OVH}$ en gatas, es la llamada "mínimamente invasiva con ovariotomo". Que consiste en un mini-abordaje por la línea media, en la cual los cirujanos usan el ovariotomo para localizar los pedículos ováricos. El tiempo promedio con esta técnica fue de 11.4 minutos (Minto et al., 2017). Estos resultados fueron corroborados en otro estudio, donde concluyen que la el uso de ovariotomo por una mini-incisión es menos invasiva y la proponen como una opción para incorporar de manera rutinaria en las 
técnicas contraceptivas veterinarias, pues presenta varias ventajas contra la celiotomía tradicional (Lawall et al., 2017).

Comparación del tiempo de cirugía laparoscópica contra cirugía abierta Durante la revisión de literatura se encontró que el tiempo de cirugía varía significativamente cuando se comparan estos dos tipos de técnicas quirúrgicas. Por ejemplo, Sakals et al. (2018), compararon la cirugía por celiotomía contra la laparoscópica. Ellos describen que el procedimiento abierto de OVH fue más rápido, pues este se realizó en un tiempo promedio 19.1 minutos, en comparación con técnicas laparoscópicas, donde en invirtieron de 27.7 a 33.2 minutos. La OVH por laparoscopia se realizó con el uso de un dispositivo sellador de vasos sanguíneos y ligaduras por sutura respectivamente, donde no obtuvieron diferencia significativa en el tiempo, no obstante ser un procedimiento más rápido el realizado con el dispositivo sellador de vasos. Sin embargo, mencionan que una mayor experiencia laparoscópica reduce considerablemente los tiempos quirúrgicos. Sobre todo, en la OV asistida, que podrá ser la mejor opción dentro de las cirugías laparoscópicas. En otro estudio, se describe una diferencia mayor, pues en la $\mathrm{OVH}$ abierta invirtieron 21 minutos en promedio contra 51.6 minutos por laparoscopia (Case et al., 2015).

En otra investigación, los tiempos de cirugía fueron estadísticamente similares en las cirugías laparoscópicas con un solo puerto y en las que usaron LigaSure ${ }^{\circledR}$ con las cirugías abiertas. Asimismo, encontraron que la ligadura con nudo extracorpóreo en laparoscopia por un solo puerto es técnicamente más difícil y consume un mayor tiempo (Coisman et al., 2014).

Comparación del tiempo entre cirugías laparoscópicas

Se han realizado estudios que comparan el tiempo de cirugía entre procedimientos contraceptivos laparoscópicos en gatas. Por ejemplo, en un estudio se comparó el uso de hemostasia por medio de electrocoagulación bipolar contra resección del pedículo con láser en OV. En este estudio, se realizó, hemostasia y se cortó uno de los pedículos con un dispositivo laser y el contralateral con uno bipolar. Estos investigadores encontraron que con el dispositivo laser el tiempo de cirugía fue estadísticamente mayor que con el bipolar; la mayor diferencia del tiempo quirúrgico ocurrió al momento de la resección del ovario, pues con el dispositivo laser tardaron $(4: 09 \pm 2: 50$ minutos), contra $(2: 23 \pm 1: 01)$ de la electrocoagulación bipolar. Además, mencionan que el abordaje al ovario derecho fue un poco más difícil, pero no interfirió en la dificultad de la cirugía (van Nimwegen y Kirpensteijn, 2007). En otro estudio donde solo realizaron OV laparoscópicas con nudo extracorpóreo, tuvieron un tiempo promedio de 44.8 minutos, el cual según los autores es similar a otros estudios donde se usó el sistema bipolar (Conceição et al., 2017). En un estudio similar, los 
investigadores encontraron que la utilización de un dispositivo hemostático bipolar reduce considerablemente el tiempo de cirugía en la OVH laparoscópica en gatas, esto cuando se compara contra un mecanismo monopolar (Howe, 2006). Existe otra investigación donde encontraron que con un dispositivo casero de laparoscopia por un solo puerto el tiempo de cirugía fue de 14.54 minutos como promedio (da Costa et al., 2017).

\section{Comparación del dolor}

El manejo del dolor durante y después de la cirugía es de los puntos más importantes a considerar en la realización de técnicas quirúrgicas contraceptivas en gatas (Murugesan et al., 2020). En este rubro no está claro cuál cirugía causa menos dolor, Por ejemplo, existe la aceptación de una falta de evidencia científica sobre si el abordaje lateral o por línea media es menos doloroso (Merritt y Collinson, 2020). De la misma manera, no se ha podido establecer si la OVH o la OV causan mayor dolor en gatas (Peeters y Kirpensteijn, 2011). También es común considerar que las técnicas laparoscópicas tienden a causar menos dolor que la cirugía tradicional (Gauthier et al., 2015).

\section{Comparación del dolor causado con las técnicas abiertas}

En la evaluación de la literatura se encontraron investigaciones que comparan el dolor producido por un abordaje tradicional por línea media con el abordaje por el flanco lateral. Se ha descrito una diferencia significativa en el dolor posquirúrgico entre ambas técnicas quirúrgicas, pues está demostrado que hay un mayor dolor cuando se usó el abordaje por flanco lateral (Burrow et al., 2005). Así mismo, existe una investigación que reporta la evidencia de mayor dolor a la palpación en las gatas con heridas del flanco lateral comparadas contra las de línea media (Coe et al., 2006).

Estos resultados contrastan con lo descrito por otros autores. Por ejemplo, Gauthier et al. (2015), no encontraron diferencias significativas en el dolor causado por las cirugías por celiotomía o laterales. Además, en otros dos estudios se menciona que, aunque existió una mayor inflamación y secreciones en las gatas con abordaje lateral, no hubo una diferencia estadísticamente significativa en cuanto al dolor posquirúrgico (Coe et al., 2006). En otro estudio encontraron resultados muy interesantes en cuanto al dolor producido con el abordaje lateral contra la celiotomía en gatas sometidas a OV. Se encontró que una hora después de la cirugía y al tiempo del alta las gatas con el abordaje lateral presentaron más dolor. Sin embargo, observaron que las gatas con OVH realizadas por la línea media presentaron más inflamación cuando fueron dadas de alta, así como a los 3 y 10 días posquirúrgicos. Interesantemente, las gatas con el abordaje lateral presentaron menos dolor a los días 3 y 10 pos-cirugía (Swaffield et al., 2020). 
Comparación del dolor causado por técnicas abiertas contra laparoscopia

Durante la revisión de literatura se encontró un artículo que describe que las gatas esterilizadas por laparoscopia mostraban escalas de dolor similares que aquellas que fueron esterilizadas por técnica abierta por línea media (Case et al., 2015). Por otro lado, Sakals et al. (2018), no se encontraron diferencias en las mediciones del dolor con escalas visual análoga en gatas con cirugía abierta contra laparoscópica. En esta investigación compararon la cirugía laparoscópica asistida usando un dispositivo bipolar, la asistida con ligadura tradicional y la abierta.

Estos resultados difieren considerablemente con los presentados por otros autores, quienes demuestran que la OVH por laparoscopia es menos dolorosa, en especial con el uso del bisturí armónico. Se demostró que las gatas con OVH tradicional presentaban mayor concentración de cortisol en sangre posquirúrgico y sus escalas de dolor eran más altas (Guedes et al., 2017). Esto está apoyado por otro estudio donde realizaron OV en gatas por laparoscopia y demostraron que es menos dolorosa que la realizada por línea media o por el flanco (Gauthier et al., 2015).

\section{Comparación del dolor causado por técnicas laparoscópicas}

Existe poca literatura en la que se compara el dolor con diferentes técnicas laparoscópicas para la OVH u OV en gatas. En un artículo no encontraron diferencias en el dolor causado por el uso de ligaduras contra un dispositivo bipolar en OV asistidas por laparoscopia (Sakals et al., 2018). Contrario a lo descrito en otra investigación, en la cual encontraron que las gatas sometidas a OV en las que ligaron por medio de nudo extracorpóreo presentaron más dolor que aquellas en donde usaron el LigaSure ${ }^{\circledR}$ (Case et al., 2015).

Tiempo de aprendizaje o dificultad técnica de cada técnica

Tiempo de aprendizaje en las técnicas abiertas

En este rubro es en el cual se encontró menos literatura científica, sin embargo, se pudieron analizar dos investigaciones: En un estudio en donde tomaron parte estudiantes del último año de veterinaria, se encontró una evolución favorable y el desarrollo de confianza durante la realización de la cirugía en un periodo de tres semanas (Freeman et al. 2017). En otra investigación en la cual se realizó una encuesta de percepción de dificultad de dos abordajes (flanco lateral y línea media) para la realización de $\mathrm{OVH}$ en gatas, los estudiantes de veterinaria determinaron que en una escala del 0 al 100, el abordaje por el flanco lateral fue más complicado con una puntación de 48, contra 41 puntos del abordaje por línea media (Coe et al., 2006).

Finalmente, no se encontraron estudios que debatan el tiempo de aprendizaje de técnicas abiertas contra laparoscópicas ni comparando técnicas laparoscópicas. 


\section{CONCLUSIONES}

En la revisión sistemática realizada en este trabajo encontramos que existe poca literatura científica con la suficiente evidencia para esclarecer la técnica quirúrgica contraceptiva más adecuada en gatas. No se logra establecer adecuadamente cual es la que causa menos dolor, es más rápida o menos difícil. Sin embargo, se pueden formar criterios importantes en algunos puntos.

En las gatas se han descrito la OVH y OV como alternativa para la cirugía contraceptiva. Actualmente no existe evidencia científica que sugiera que una técnica es superior a otra y ambas pueden realizarse por un abordaje por línea media, flanco lateral o por laparoscopia. La ovariectomía es una alternativa poco usada pero cuyas ventajas (menor tiempo de cirugía, incisiones más pequeñas y evitar posibles complicaciones uterinas) son mayores a sus desventajas.

El tiempo de cirugía es similar en los dos abordajes abiertos (lateral y celitomía). Sin embargo, la miniceliotomía con ovariotomo resultó ser la técnica más rápida. El procedimiento de la ligadura del pedículo ovárico es el paso más lento; el tiempo de este se reduce con el uso de nudo pedicular. La utilización de un sistema de electrocoagulación bipolar no mostró reducir el tiempo en cirugías abiertas en las gatas, contrario a lo ocurrido en perras.

La cirugía laparoscópica es más lenta y requiere de una mayor curva de aprendizaje, sin embargo, con práctica y el uso de dispositivos especiales (LigaSure®) los tiempos son muy similares a los obtenidos por cirugía abierta. Además, con esta técnica el uso de electrobisturí bipolar y el bisturí armónico por coagulación ultrasónica sí reducen el tiempo de cirugía.

En cuanto al dolor ocasionado en los dos abordajes para practicar la cirugía abierta, no se ha establecido una superioridad de alguno de ellos. Aunque el abordaje por línea media causa menos dolor en las primeras 24 horas, las gatas con abordaje lateral presentaron menos dolor después de 72 horas. También se ha establecido que la cirugía laparoscópica causa menos dolor que la técnica abierta.

\section{LITERATURA CITADA}

ADIN, CA. 2011. Complications of ovariohysterectomy and orchiectomy in companion animals. Veterinary Clinics of North America - Small Animal Practice. 41(5):1023-39. http://dx.doi.org/10.1016/..cvsm.2011.05.004

BEGUM M, Bhuvaneshwari V. 2018. Single suture ovariohysterectomy-a novelminimally invasive surgical technique in dogs and cats. International Journal of Recent Scientific Research. 9(12):30098-30099. http://dx.doi.org/10.24327/ijrsr.2018.0912.2985 
BOHLING MW. 2020. Complications in spay and neuter surgery. En: White S high-quality, high-volume spay and neuter and other shelter surgeries. Hoboken, NJ, USA: John Wiley \& Sons. Pp. 339-61. https://doi.org/10.1002/9781119646006.ch17

BOURSIER JF, Bassanino J, Leperlier D. 2018. Effectiveness of a bipolar vessel sealant device for ovariohysterectomy in cats with pyometra. Journal of Feline Medicine and Surgery. 20(12): 1119-1123. https://doi.org/10.1177/1098612X17752581

BURROW R, Batchelor D, Cripps P. 2005. Complications observed during and after ovariohysterectomy of 142 Bitches at a veterinary teaching hospital. Veterinary Record. 157(26): 829-33. http://dx.doi.org/10.1136/vr.157.26.829

BUSHBY P, White S. 2019. Dog spay/cat spay. En: White S High-Quality, High-Volume Spay and Neuter and Other Shelter Surgeries. Hoboken, NJ, USA: John Wiley \& Sons. Pp. 239-65. https://doi.org/10.1002/9781119646006.ch12

CASE J. Boscan PL, Monnet EL, Niyom SR, Imhoff DJ, Wallace ML, SmeakDD 2015. Comparison of surgical variables and pain in cats undergoing ovariohysterectomy, laparoscopic-assisted ovariohysterectomy, and laparoscopic ovariectomy. Journal of the American Animal Hospital Association. 51(1): 1-7. https://doi.org/10.5326/JAAHA-MS5886

COE RJ, Grint NJ, Tivers MS, Moore AH, Holt E. 2006. Comparison of flank and midline approaches to the ovariohysterectomy of cats. Veterinary record. 159(10): 309313. http://dx.doi.org/10.1136/vr.159.10.309

COISMAN JG, Case JB, Shih A, Harrison K, Isaza N, Ellison G. 2014. Comparison of surgical variables in cats undergoing single-incision laparoscopic ovariectomy using a ligasure or extracorporeal suture versus open ovariectomy. Veterinary Surgery. 43(1): 38-44. https://doi.org/10.1111/j.1532-950X.2013.12073.x

CONCEIÇÃO MEBA, Mariano RSG, Crivelaro RM, Silva MAM, de Moraes PC, Teixeira PPM, Dias LGGG. 2017. Description and executability of a novel pre-tied mini ligature (Miniloop) in laparocopic ovariectomy in cats. Acta Scientiae Veterinariae. 45:1-6. http://www.ufrgs.br/actavet/45/PUB\%201492.pdf

CONCEIÇÃO MEBA, Uscategui RAR, Bertolo PHL, de Souza DC, Rolemberg DDS, de Moraes PC, Teixeira PPM, Dias LGGG. 2018. Assessment of postoperative inflammatory markers and pain in cats after laparoscopy and miniceliotomy ovariectomy. Veterinary Record. 183(21): 656. http://dx.doi.org/10.1136/vr.104776

CUDDY LC. 2016. Spay/neuter - What's the evidence?. Veterinary Ireland Journal. 7(7): 351-352. http://www.veterinaryirelandjournal.com/images/pdf/focus/focus1_jul_2017.pdf 
DA COSTA D, Libardoni RN, Schmitt JT, Padilha AS, Schulz Jr FJorge, Ataíde MW, Menezes FB, Allievi K, Brun M V, Teixeira PPM, Silva MAM. 2017. LESS ovariohysterectomy in cats using a new homemade multiport. Ciência Rural. 47(11): 20161130. https://dx.doi.org/10.1590/0103-8478cr20161130

DeTORA M, McCarthy RJ. 2011. Ovariohysterectomy versus ovariectomy for elective sterilization of female dogs and cats: is removal of the uterus necessary? Journal of the American Veterinary Medical Association. 239(11): 1409-12. https://dx.doi.org/10.2460/javma.239.11.1409

FRASSON BA. 2018. Urogenital system. Ovaries and uterus. In Veterinary Surgery: Small Animal. Vol. 2, ed. Karen Johnston, Spencer, Tobias. St. Louis, Missouri: Elsevier, 2109-29. ISBN: 9780323320658

FREEMAN LJ, Ferguson N, Fellenstein C, Johnson R, Constable PD. 2017. Evaluation of learning curves for ovariohysterectomy of dogs and cats and castration of dogs. Journal of the American Veterinary Medical Association. 251(3): 322-332. https://doi.org/10.2460/javma.251.3.322

GAUTHIER O, Holopherne-Doran D, Gendarme T, Chebroux A, Thorin C, Tainturier D, Bencharif D. 2015. Assessment of postoperative pain in cats after ovariectomy by laparoscopy, median celiotomy, or flank laparotomy. Veterinary Surgery. 44(S1): 23-30. https://doi.org/10.1111/j.1532-950X.2014.12150.x

GRIFFIN B, Bushby PA, McCobb E, White SC, Rigdon-Brestle KY, Appel LD, Makolinski KV, Wilford CL, Bohling MW, Eddlestone SM, Farrell KA, Ferguson N, Harrison K, Howe LM, Isaza NM, Levy JK, Looney A, Moyer MR, Robertson SA, Tyson K. 2016. The Association of Shelter Veterinarians' 2016 veterinary medical care guidelines for spay-neuter programs. Journal of the American Veterinary Medical Association. 249(2): 165-188 https://doi.org/10.2460/javma.249.2.165

GUEDES RL, de Souza FW, Freitas GC, Gomes, Castro JL, Marconato F, Castro VSP, Pippi NL, Dornbusch PT. 2017. Evaluation of electrosurgery and titanium clips for ovarian pedicle haemostasis in video-assisted ovariohysterectomy with two portals in bitches. Ciencia Rural. 47(2): 2-7. https://doi.org/10.1590/0103-8478cr20151245

HILL LN, Smeak DD. 2010. Suspensory ligament rupture technique during ovariohysterectomy in small animals. Compendium: Continuing Education For Veterinarians. 32(6): E1-E7.

https://www.researchgate.net/profile/Lawrence_Hill/publication/47430864_Surgical_view s_suspensory_ligament_rupture_technique_during_ovariohysterectomy_in_small_anim als/links/0046351edc92ebeed0000000.pdf 
HOWE LM. 2006. Surgical methods of contraception and sterilization. Theriogenology. 66(3 SPEC. ISS.): 500-509. DOI: 10.1016/j.theriogenology.2006.04.005

KIANI FA, Kachiwal AB, Shah MG, Nizamani ZA., Khand FM, Lochi GM, Haseeb A, Khokhar AM, Oad A, Ansari MI. 2014. Comparative study on midline and flank approaches for ovariohystrectomy in cats. Journal of Agriculture and Food Technology. 4(2): 21-31. $\quad$ https://www.researchgate.net/profile/GhulamLochi/publication/263620334_Comparative_Study_on_Midline_and_Flank_Approaches for_Ovariohystrectomy_in_Cats/links/00b4953b652438caf4000000/Comparative-Studyon-Midline-and-Flank-Approaches-for-Ovariohystrectomy-in-Cats.pdf

LAWALL T, Beck CAC, Queiroga LB, dos Santos FR. 2017. Minilaparoscopic ovariohysterectomy in healthy cats. Ciência Rural. 47(2): e20160261. https://doi.org/10.1590/0103-8478cr20160261

MANTEROLA DC, Zavando D. 2009. Cómo interpretar los niveles de evidencia en los diferentes escenarios clínicos. Revista Chilena de Cirugia. 61(6): 582-95. http://dx.doi.org/10.4067/S0718-40262009000600017

MAYHEW PD, Brown DC. 2007. Comparison of three techniques for ovarian pedicle hemostasis during laparoscopic-assisted ovariohysterectomy. Veterinary Surgery. 36(6): 541-47. https://doi.org/10.1111/j.1532-950X.2007.00280.x

MC GRATH H, Hardie RJ, Davis E. 2004. Lateral flank approach for ovariohysterectomy in small animals. Compendium on Continuing Education for the Practicing Veterinarian. 26(12): $\quad 922-30 . \quad$ https://vetfoliovetstreet.s3.amazonaws.com/mmah/09/4b8f4bfa764cc9b20481c797956733/filePV_26_ 12_922.pdf

MERRITT B, Collinson A. 2020. Midline versus flank approach for spaying cats-is one less painful than the other? The Veterinary Record. 186(17): 565-67. https://doi.org/10.1136/vr.m2008

MILLER KP, Rekers W, Ellis K, Ellingsen K, Milovancev M. 2016. Pedicle ties provide a rapid and safe method for feline ovariohysterectomy. Journal of Feline Medicine and Surgery. 18(2): 160-64. https://doi.org/10.1177/1098612X15576589

MINTO BW, Brondani JT, Giordano T, Prada TC, 1, Luna SPL, Gianuzzi LL, Nicole W. Pereira NW,.Brandão CVS. 2017. Spay hook minimally invasive ovariohysterectomy in cats. Retrospective study of 276 cases. Investigação. 16(2): 29-33.

https://publicacoes.unifran.br/index.php/investigacao/article/view/2354

MURARO L, White RS. 2014. Complications of ovariohysterectomy procedures performed in 1880 dogs. Tierarztliche Praxis Ausgabe K: Kleintiere - Heimtiere. 42(5): 297-302. https://doi.org/10.1055/s-0038-1623776 
MURUGESAN V, Arunachalam K, Shanmugam K, Palanivel M. 2020. Post-operative behavioural assessment of cats undergoing ovariohysterectomy. International Journal of Current Microbiology and Applied Sciences. 9(7): 609-614. https://doi.org/10.20546/ijcmas.2020.907.068

OVERLEY B, Shofer FS, Goldschmidt MH, Sherer D, Sorenmo KU. 2005. Association between ovarihysterectomy and feline mammary carcinoma." Journal of Veterinary Internal Medicine. 19(4):560-563.

https://doi.org/10.1892/0891-6640(2005)19[560:aboafm]2.0.co;2

PEETERS ME, Kirpensteijn K. 2011. Comparison of surgical variables and short-term postoperative complications in healthy dogs undergoing ovariohysterectomy or ovariectomy. Journal of the American Veterinary Medical Association. 238(2): 189-94. https://doi.org/10.2460/javma.238.2.189

PEREIRA MAA. Gonçalves LA, Evangelista MC, Thurler RS, Campos KD, Formenton MR, Patricio GCF, Matera JM, Ambrósio AM, Fantoni DT. 2018. Postoperative pain and short-term complications after two elective sterilization techniques: ovariohysterectomy or ovariectomy in cats. BMC Veterinary Research. 14(1): 335. https://dx.doi.org/10.1186/s12917-018-1657-z

PHYPERS C. 2017. In cats and dogs does laparoscopic ovariectomy offer advantages over open ovariectomy for postoperative recovery? Veterinary Evidence. 2(2):1-14. http://dx.doi.org/10.18849/ve.v2i2.59

POLLARI FL. Bonnett BN, Bamsey SC, Meek AH, Allen DG. 1996. Postoperative complications of elective surgeries in dogs and cats determined by examining electronic and paper medical records. Journal of the American Veterinary Medical Association. 208(11):1882-6. https://pubmed.ncbi.nlm.nih.gov/8675479/

PORTERS N. Polis I, Moons C, Duchateau L, Goethals K, Huyghe S, De Rooster H. 2014. Prepubertal gonadectomy in cats: different surgical techniques and comparison with gonadectomy at traditional age. Veterinary Record. 175(9):223. http://dx.doi.org/10.1136/vr.102337

REECE JF. 2018. Ovariohysterectomy-flank approach. En: Polak K, Kommedal AT, Field Manual for Small Animal Medicine. Hoboken, NJ, USA: John Wiley \& Sons. Pp. 229-236. https://doi.org/10.1002/9781119380528.ch9b

SACKETT DL, Wennberg JE. 1997. Choosing the best research design for each question. British Medical Journal. 315(7123): 1636-1636. https://doi.org/10.1136/bmj.315.7123.1636 
SAKALS SA. Rawlings CA, Laity J, Hofmeister EH, Radlinsky MG. 2018. Evaluation of a Laparoscopically assisted ovariectomy technique in cats. Veterinary Surgery. 47(S1):O32-O38. http://dx.doi.org/10.1111/vsu.12762

SILVA-MOLANO RF, Grajales-Galindo NL, Mejía-Henao RA, Loaiza-Echeverri AM. 2007. Evaluación de ovariectomía mediante abordaje paracostal y angiotripsia , como método de esterilización en caninos evaluation of ovariectomy by means of boarding paracostal and angiotripsy, as method of sterilization in canine. Veterinária e Zootecnia. 1(1): 29-35. http://vip.ucaldas.edu.co/vetzootec/downloads/v1n1a05.pdf

STAVISKY J, Marnie B. 2020. Comparing wound complications associated with midline and flank approaches for spaying cats. Veterinary Record. 186(6). https://doi.org/10.1136/vr.m532

SWAFFIELD MJ, Molloy SL, Lipscomb VJ. 2020. Prospective comparison of perioperative wound and pain score parameters in cats undergoing flank vs midline ovariectomy. Journal of Feline Medicine and Surgery. 22(2):168-177. https://doi.org/10.1177/1098612X19837038

VAN NIMWEGEN SA., Kirpensteijn J. 2007. Laparoscopic ovariectomy in cats: comparison of laser and bipolar electrocoagulation. Journal of Feline Medicine and Surgery. 9(5): 397-403. https://doi.org/10.1016\%2Fj.jfms.2007.03.007

VOORWALD FA, Tiosso CF, Toniollo GH. 2013. Gonadectomia pré-puberal em cães e gatos tt - prepubertal gonadectomy in dogs and cats. Ciência Rural. 43(6): 1082-91. https://doi.org/10.1590/S0103-84782013005000059

WATTS J. 2018. The use of bipolar electrosurgical forceps for haemostasis in open surgical ovariectomy of bitches and queens and castration of dogs. Journal of Small Animal Practice. 59(8): 465-473. https://doi.org/10.1111/jsap.12838

YATES D, Goetz U. 2015. A step-by-step guide to the pregnant cat spay. Companion Animal. 20(7): 394-398. https://doi.org/10.12968/coan.2015.20.7.394 